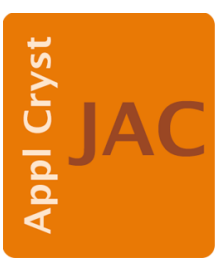

JOURNAL OF

APPLIED

CRYSTALLOGRAPHY

Volume 54 (2021)

Supporting information for article:

Combined specular and off-specular reflectometry: elucidating the complex structure of soft buried interfaces

Aljoša Hafner, Philipp Gutfreund, Boris P. Toperverg, Andrew O. F. Jones, Johann P. de Silva, Andrew Wildes, Henry E. Fischer, Mark Geoghegan and Michele Sferrazza 


\title{
Supporting Information: Combined Off-specular and Specular reflectometry: Elucidating the complex structure of soft buried interfaces
}

Aljoša Hafner, ${ }^{a, b}$, Philipp Gutfreund ${ }^{a}$, Boris P. Toperverg ${ }^{a, c}$, Andrew O.F. Jones ${ }^{b}$, Johann P. de Silva ${ }^{b}$, Andrew Wildes ${ }^{a}$, Henry E. Fischer ${ }^{a}$, Mark Geoghegan ${ }^{d}$, Michele Sferrazza $a^{b}$

\author{
${ }^{a}$ Institut Laue - Langevin, 71 avenue des Martyrs, CS 20156, 38042 Grenoble, Cedex 9, France \\ ${ }^{~ '}$ Université Libre de Bruxelles, Département de Physique, Boulevard du Triomphe, 1050 Brussels, Belgium \\ 'Petersburg Nuclear Physics Institute, National Research Center "Kurchatov Institute", 188300 Gatchina, Russia \\ ${ }^{d}$ Department of Physics and Astronomy, University of Sheffeld, Hicks Building, Hounsfield Road, Sheffield S3 7RH, United
}

Kingdom

\section{Decomposition of optical enhancements in OSS maps}

A scope of features in Fig. 4 can be understood analysing different contributions stemming from the transverse form-factor $G_{11}^{\perp}$ in Eq. 45. Indeed, substitution of Eq.(42) into Eq.(45) yields the explicit equation,

$$
\begin{aligned}
G_{l l}^{\perp} & =\left|t_{l}^{f} t_{l}^{i}+\widetilde{r}_{l}^{f} \widetilde{r}_{l}^{i}\right|^{2}\left|F_{l}\right|^{2}+\left|t_{l}^{f} \widetilde{r}_{l}^{i}+\widetilde{r}_{l}^{f} t_{l}^{i}\right|^{2}|\widetilde{F}|^{2} \\
& +2 \Re\left\{\left\{\left(t_{l}^{f} t_{l}^{i}+\widetilde{r}_{l}^{f} \widetilde{r}_{l}^{i}\right)\left(t_{l}^{f} t_{l}^{i}+\widetilde{r}_{l}^{f} \widetilde{r}_{l}^{i}\right)^{*} F_{l} \widetilde{F}^{*}\right\},\right.
\end{aligned}
$$

in which $G_{l l}^{\perp}$ is represented as a sum of three terms of different symmetry. These terms are plotted in a subset of three maps in Fig. S1.The contribution of the first term into the sum reaches maximum values along the lines $p_{f, i}=2 \pi n / d_{1}-p_{i, f}$ seen in Fig. $S 1$ as a periodic sequence of ripples at integer $n \geq$ $p_{i} d_{1} f(2 \pi)$. Intensities of ripples are almost constant along each of their crests, while decreasing with the order $n$ at $p_{i} \geq p_{c 1}$, or $p_{f} \geq p_{c 1}$. Their line shape becomes distorted approaching positions of critical $p_{i} \approx p_{c 2}$, or $p_{f} \approx p_{c 2}$, while intensities sharply decrease at $p_{i} \leq p_{i 2}$, or $p_{f} \leq p_{i 2}$. In contrast, the second term in Eq.(S 1) reveals periodicity in the orthogonal direction, i.e. along the lines $\left|p_{f}-p_{i}\right|=2 \pi n / d_{1}$ manifested in Fig. $S 1$ by a set of ridges running at integer $n$ parallel to the main diagonal at $n=0$. The latter traces the SR ridge (not included). Intensities along ridges reach maximum values when intersecting the vertical lines $p_{i} \approx p_{c 2}$, or horizontal lines, $p_{f} \approx p_{c 2}$, while decaying along ridges with the increasing sum $\left(p_{f}+p_{i}\right)$ if $p_{i} \geq p_{c 2}$ and $p_{f} \geq p_{c 2}$. Below the critical edges $p_{c 1}$ for h-PMMA the intensities of ridges abruptly drop to zero and steadily decrease with increasing of orders $n$.

Fig. S1 illustrates the contribution of the third term in Eq.(S 1). As this pattern originates from the cross-term of the scattering functions it may become negative and is therefore plotted in linear scale. Such easy interpretation of various features in Fig. 3 is possible when data are collected as a function of angles of incidence, $\alpha_{i}$, and scattering, $\alpha_{f}$, at fixed wavelength $\lambda$ which just plays a role of a scaling parameter unique for both axes $p_{i}$ and $p_{f}$.

In contrast, the visual inspection and interpretation of data collected in ToF mode as a function of $\lambda$ and $\alpha_{f}$ at fixed $\alpha_{i}$, although containing about the same information as the $2 \mathrm{D}$ map above, requires some efforts. An example of the 2D map calculated for exactly the same model, but presented in the coordinates $\alpha_{f}$ and $\lambda$ at a fixed value of $\alpha_{i}$ is depicted in Fig. S2 for the same sum of partial transverse form-factors plotted in Fig.4. Next, three maps in Fig. 33 illustrate different ingredients composing the map in Fig. S2 in the same way as maps in Fig. S1 compose the map in Fig. 4. Comparing Figs. S1 and S3 one can see that, only the middle and bottom panels contain an intensity line located on the SR ridge, which is now running vertically at $\alpha_{f}=\alpha_{i}$. Other OSS features cannot be explained without comprehensive analysis. Among those features one may, however, recognize the ranges of total reflection of incident, or/and scattered neutron waves introducing critical wavelengths $\lambda_{c l}^{i}\left(\alpha_{i}\right)=$ $\lambda_{0 l} \sin \alpha_{i}$ and $\lambda_{c l}^{f}\left(\alpha_{f}\right)=\lambda_{0 l} \sin \alpha_{f}$, where $\lambda_{l}=2 \pi / p_{c l}$ is the wavelength of total reflection at normal incidence onto the $t^{\text {th }}$ layer. For a silicon substrate $\lambda_{o s} \approx 1200 \AA$, and for $\alpha_{i}=1^{\circ}$ the critical wavelength is $\lambda_{c s}=21 \AA$. Hence, if $\lambda$ exceeds this value, but is still below the critical wavevector for h-PMMA, the neutron wave is totally reflected from the Si substrate, and interferes with the incident wave in the thick h-PMMA layer and thus scattering in OSS directions.

Hence, the set of maps in Fig. S3 is, qualitatively, not so useful as that in Fig. 33 , due to the fact that in the $\lambda$ vs. $\alpha_{f}$ representation the symmetry of Fig. S1 intimately related with the reciprocity principle is lost. Indeed, Eq.(45) and hence its components in Eq.(S 1) are explicitly invariant with respect to interchange between $p_{f}$ and $p_{i}$, but this does not necessarily mean they obey the reciprocity principle. The latter requires invariance with respect to the interchange between $\alpha_{f}$ and $\alpha_{i}$ at each fixed value of $\lambda$, if scattering is elastic. 


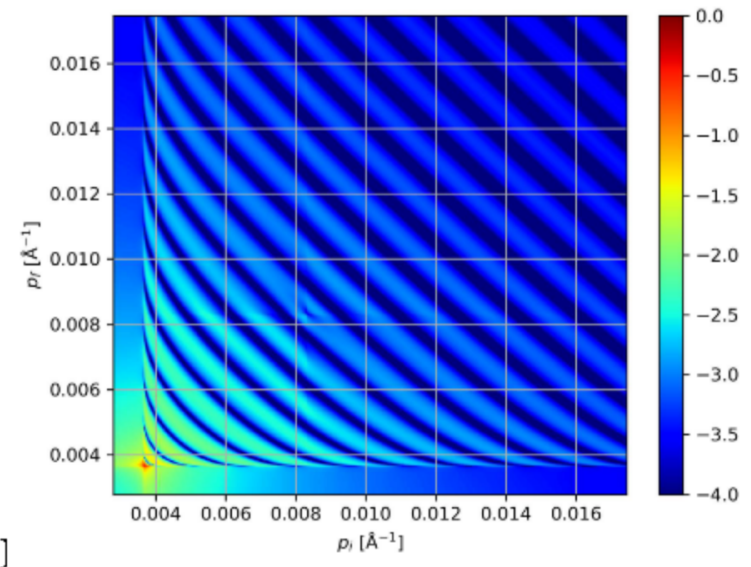

[a]

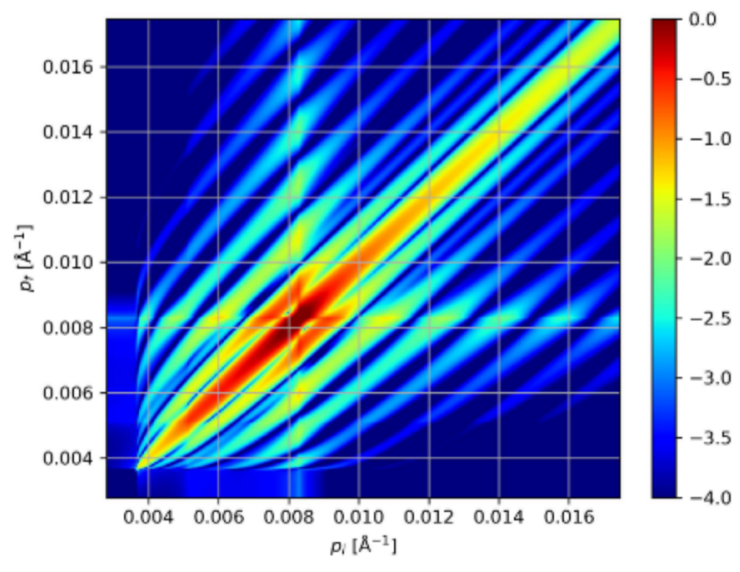

[b]

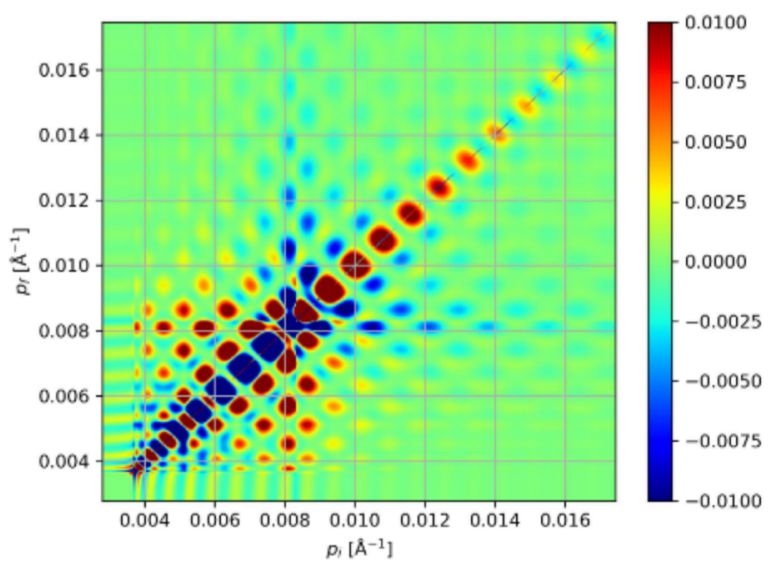

\section{Figure S1}

Different contributions composing the map in Fig. 4. The maps correspond to the three terms in Eq. 45: a) First term, b) second term and c) third term.

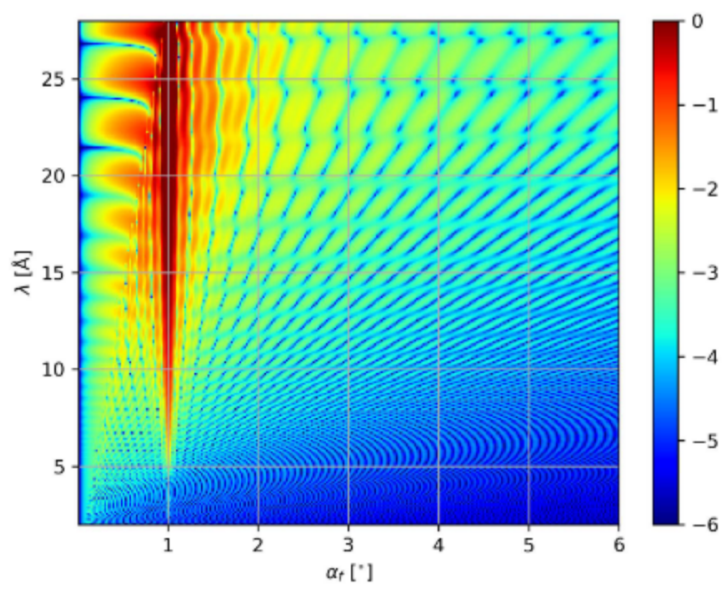

Figure S2

The same function as in Fig. 4, but plotted as a function of the angle of scattering $x_{f}$ and the wavelength $\lambda$. 

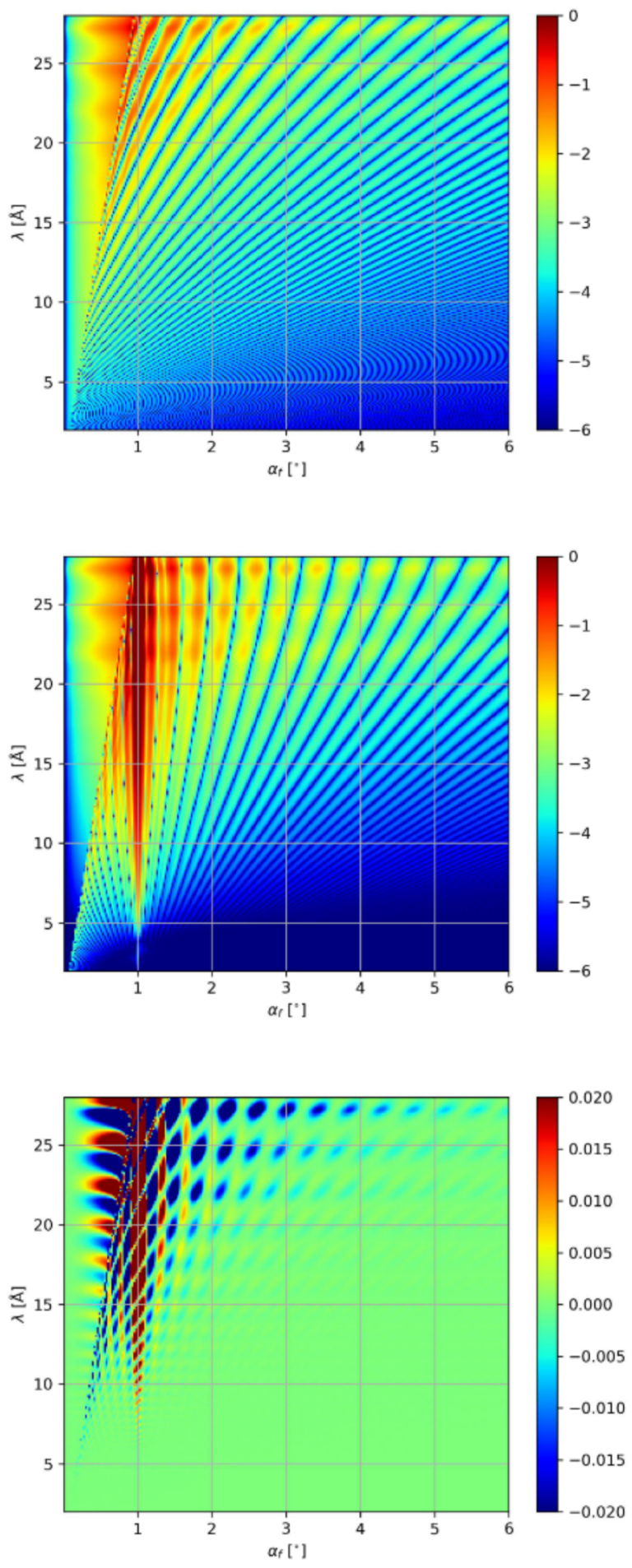

\section{Figure S3}

The same data as in Fig. S1, but plotted as a function of the angle of scattering $\alpha f_{f}$ and the wavelength $\lambda$.

\section{Concentration fluctuations in binary compounds}

Let us consider a simplest, but relevant to our experimental situation, case when one of the layers of the multilayer stack contains some amount $\mathcal{N}_{l}$ of inclusions which SLD $\rho_{l}^{A}$ differs from the surrounding matrix SLD $\rho_{l}^{B}$. Let us also suppose that inclusions are of a shape of right cylinders with their axes nor$\mathrm{mal}$ to the interfaces and heights determined by the layer thickness $d_{l} \ll \mathcal{R}_{l}^{i}$, where $\mathcal{R}_{l}^{i}$ refers to in-plane dimensions of cylinders numerated over the layer $l$ plane by the superscript $i$. The base area $s_{l}^{i}$ of cylinders is restricted by the closed contour line $\mathcal{R}_{l}^{i}\left(\omega_{i}\right)$ depending on the polar angle $\omega$ of the $2 \mathrm{D}$ radius vector $\left(\boldsymbol{r}_{\|}-\boldsymbol{r}_{\|}^{i}\right)$, where the vector $\boldsymbol{r}_{\|}^{i}$ determines the cylinder position. Inside the contour, i.e. at $\left|\boldsymbol{r}_{\|}-\boldsymbol{r}_{\|}^{i}\right| \leq \mathcal{R}_{l}^{i}$, the SLD is equal to $\rho_{l}=\rho_{l}^{A}$, while $\rho_{l}=\rho_{l}^{B}$ elsewhere over the layer $l$. Note, that if inclusion's material is immiscible with that of the polymer matrix then the cylinder's base has preferably circular shape with radius $\mathcal{R}_{i}^{i}$ independent of $\omega_{i}$.

For such system the in-plane SLD distribution $\rho\left(\boldsymbol{r}_{\|}\right)$, following to Ref.(Krivoglaz, 1969), is described by the sum:

$$
\rho\left(\boldsymbol{r}_{\|}\right)=\left(\rho^{A}-\rho^{B}\right) \sum_{i=1}^{N_{3}} \mathcal{F}_{l}^{i}\left(\boldsymbol{r}_{\|}-\boldsymbol{r}_{\|}^{i}\right)+\rho^{B},
$$

where the in-plane shape function $\mathcal{F}_{l}^{i}\left(\boldsymbol{r}_{\|}-\boldsymbol{r}_{\|}^{i}\right)=1$, if $\left|\boldsymbol{r}_{\|}-\boldsymbol{r}_{\|}^{i}\right| \leq$ $\mathcal{R}_{l}^{i}$ and $\mathcal{F}_{l}^{i}\left(\boldsymbol{r}_{\|}-\boldsymbol{r}_{\|}^{i}\right)=0$ otherwise.

Eq.(S 2) can be readily averaged over the layer plane providing the following expression for the mean value of SLD:

$$
\left\langle\rho_{l}\right\rangle=c_{l}\left(\rho_{l}^{A}-\rho_{l}^{B}\right)+\rho_{l}^{B},
$$

where $c_{l}=S_{l}^{A} / S_{\text {OSS }}$ is the concentration of inclusions, and $S_{l}^{A}=\sum_{i}^{N_{j}} s_{l}^{i}$ is the total base area of all cylinders illuminated by the beam.

Taking into account Eq.(S 2) and Eq.(S 3) the lateral factor for the layer $l$ in Eq.(41) can be rewritten as:

$$
\Delta \rho_{l}\left(\boldsymbol{q}_{\|}\right)=\left(\rho_{l}^{A}-\rho_{l}^{B}\right) \sum_{i=1}^{N_{3}}\left\{e^{-i \boldsymbol{q}_{\|} \boldsymbol{r}_{\|}^{i}} F_{l}^{i}\left(\boldsymbol{q}_{\|}\right)-F_{l}^{i}(\mathbf{0})\right\} .
$$

Here the cylinder form-factor $F_{l}^{i}\left(\boldsymbol{q}_{\|}\right)$is the 2D Fourier transform,

$$
F_{l}^{i}\left(\boldsymbol{q}_{\|}\right)=\int d \boldsymbol{r}_{\|} e^{-i \boldsymbol{q}_{\|} \boldsymbol{r}_{\|}} \mathcal{F}_{l}^{i}\left(\boldsymbol{r}_{\|}\right)
$$

of the lateral shape function of the cylinder, while $F_{l}^{i}(0)=s_{l}^{i}$ is its base area such that the sum over all $i$ is equal to the total area $S_{l}^{A}=c_{l} S_{\text {OSS }}$.

It is important to remind that, due to the last term in Eq.(S 4) the scattering amplitude in Eq.(40) is nonzero only if $\boldsymbol{q}_{\|} \neq 0$.

That is why the pair correlator $G_{l l}^{\|}\left(\boldsymbol{q}_{\|}\right)$in Eq.(46) obviously turns into zero at $\boldsymbol{g}_{\|}=0$ and does not contribute to the SR. If, alternatively, $\boldsymbol{q}_{\|} \neq 0$ then from Eq.(S 4) it follows that,

$$
G_{l l}^{\|}\left(\boldsymbol{q}_{\|}\right)=\left|\rho_{l}^{A}-\rho_{l}^{B}\right|^{2} \sum_{i, j=1}^{N_{j}}\left\langle F_{l}^{i}\left(\boldsymbol{q}_{\|}\right) F_{l}^{j}\left(-\boldsymbol{q}_{\|}\right) e^{-i \boldsymbol{q}_{\|}\left(\boldsymbol{r}_{\|}^{\prime}-\boldsymbol{r}_{\|}^{j}\right)}\right\rangle
$$


Angular brackets in this equation denote two types of in-plane averaging: the first running over different shapes and sizes of cylinders, and the second one running over distances $a_{l}=\mid r_{\|}^{i}-$ $r_{\|}^{j} \mid$ between their positions. Assuming that $a_{l} \ll \mathcal{R}_{l}$ these two types averaging are often accomplished independently. Then the correlator is conveniently represented as a sum of two terms,

$$
G_{l l}^{\|}\left(\boldsymbol{q}_{\|}\right)=G_{l l}^{\mathrm{inc}}\left(\boldsymbol{q}_{\|}\right)+G_{l l}^{\mathrm{coh}}\left(\boldsymbol{q}_{\|}\right)
$$

where the first term contains only diagonal elements with $j=i$ and therefore survives for totally random distribution of inclusion positions, while the second one takes into account correlations between these positions.

Hence the first term,

$$
G_{l l}^{\mathrm{inc}}\left(\boldsymbol{q}_{\|}\right)=\mathcal{N}_{l}\left|\rho_{l}^{A}-\rho_{l}^{B}\right|^{2} c_{l}\left(1-c_{l}\right)\left\langle\left|F_{l}^{\|}\left(\boldsymbol{q}_{\|}\right)\right|^{2}\right\rangle
$$

is expressed via the auto-correlator $\left\langle\left|F_{l}^{i}\left(\boldsymbol{q}_{\|}\right)\right|^{2}\right\rangle$ and determined by the mean value of inclusion dimensions $\mathcal{R}$. If this value is small then the corresponding diffuse scattering spreads into a relatively large solid angle and may be seen in OSS as a flat background modulated by the optical effect. However it may be well detectable via GISANS.

The second term takes into account interference between the scattering from different inclusions and can be written as follows:

$$
G_{l l}^{\mathrm{coh}}\left(\boldsymbol{q}_{\|}\right)=\mathcal{N}_{l}\left|\rho_{l}^{A}-\rho_{l}^{B}\right|^{2}\left|\left\langle\mid F_{l}\left(\boldsymbol{q}_{\|}\right)\right\rangle\right|^{2} \varepsilon\left(q_{\|}\right)
$$

via the positions correlator,

$$
\varepsilon\left(q_{\|}\right)=\frac{1}{\mathcal{N}_{l}} \sum_{i \neq j}^{N_{i}}\left\langle e^{-i q_{\|}\left(\boldsymbol{r}_{\|}^{i}-r_{\|}^{j}\right)}\right\rangle .
$$

In many cases the latter can be approximated by the Lorentzian squared (in case of exponential decay in 3D), or a simple Lorentzian with the correlation length $\xi_{l}$, as above in Eq.(50) and done in this experimental work.

\section{Typical AFM micrograph for advanced stage of dewetting of the Case 1 sample}

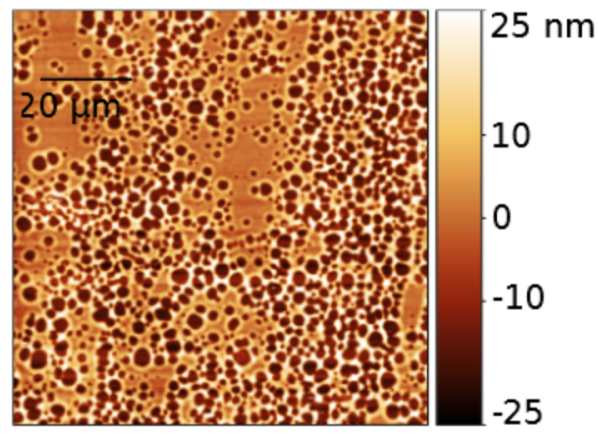

\section{Figure S4}

AFM topography of the final state of the DW4 bilayer sample.

\section{All fitting parameters for SR and OSS}

Instability of the top layer, Sample DW4 (Case A) All fitting parameters for SR and OSS for the Case A sample.

Table S1

\begin{tabular}{|c|c|c|c|c|c|c|c|c|c|c|c|}
\hline $\begin{array}{c}\hat{l}_{\text {anth }} \\
{[\mathrm{min}]}\end{array}$ & $\begin{array}{c}d_{\mathrm{d}-\mathrm{PS}} \\
{[\AA]}\end{array}$ & $\begin{array}{c}d_{\text {inter }}^{x} \\
{[\AA]} \\
{[\AA]}\end{array}$ & 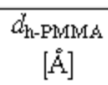 & $\begin{array}{c}\rho_{\mathrm{h}-\mathrm{PMM}} \\
{\left[\begin{array}{lll}10^{6} & 6 & \AA^{2}\end{array}\right]}\end{array}$ & $\begin{array}{c}\rho_{\mathrm{d}-\mathrm{PS}} \\
{\left[\begin{array}{lll}10 & { }^{6} \AA & { }^{2}\end{array}\right]}\end{array}$ & {$\left[\begin{array}{lll} & \rho_{\text {inter }} & \\
& 6 & \AA\end{array}\right]$} & $\begin{array}{c}\sigma_{\text {inter }} \\
{[\AA]}\end{array}$ & $\begin{array}{c}\xi_{1} \\
{[\mu \mathrm{m}]}\end{array}$ & $\begin{array}{c}\xi_{2} \\
\lfloor\mu \mathrm{m}]\end{array}$ & $\begin{array}{c}\xi_{3} \\
{[\mu \mathrm{m}]}\end{array}$ & $\begin{array}{c}c_{h} \\
{[\%]}\end{array}$ \\
\hline 0 & $155(1)$ & 0 & $1700(3)$ & $0.91(2)$ & $6.43(3)$ & - & - & - & $1.8(2)$ & - & - \\
\hline 10 & $154(1)$ & 0 & $1680(3)$ & $1.01(2)$ & $6.11(1)$ & - & - & - & $1.9(3)$ & - & - \\
\hline 15 & $151(1)$ & $10(3)$ & $1646(3)$ & $1.11(2)$ & $6.28(3)$ & 3.7 & $40(2)$ & $1.2(2)$ & $2.3(5)$ & $0.2(5)$ & $6.0(5)$ \\
\hline 20 & $149(1)$ & $18(1)$ & $1643(4)$ & $1.10(2)$ & $6.1(1)$ & $3.7(3)$ & $35(1)$ & $5(1)$ & $1.8(7)$ & $0.4(4)$ & $7.0(5)$ \\
\hline 25 & $151(51)$ & $11(4)$ & $1646(4)$ & $1.16(2)$ & $6.05(3)$ & $4.6(4)$ & $35(2)$ & $12.0(5)$ & $2.4(5)$ & $0.2(5)$ & $11(1)$ \\
\hline 30 & $153(14)$ & $7(2)$ & $1629(2)$ & $1.06(2)$ & $6.09(2)$ & $4.2(2)$ & $50(2)$ & $18(1)$ & $1.5(3)$ & $0.4(5)$ & $9(1)$ \\
\hline 40 & $155(19)$ & $3(2)$ & $1632(2)$ & $1.06(2)$ & $6.1(1)$ & $3.1(2)$ & $58(2)$ & $19(1)$ & $0.6(5)$ & $0.6(5)$ & $11(1)$ \\
\hline
\end{tabular}

Fitted structural parameters for sample DW/ as a function of annealing time. The errors are from the fit. Parameters $d_{\text {inter }}, \sigma_{\text {inter }}$ and $\rho_{\text {inter }}$ together form an artificial layer at the interface, thus extending the interface into the top $\mathrm{h}$-PMMA layer. In the late stages of annealing, the parameters $\xi_{2}$ and $\xi_{3}$ are both describing a gradual contrast between the top $\mathrm{h}-\mathrm{PMML}$ and bottom d-PS layer. The respective fits are shown in figure 8.

\begin{tabular}{|c|c|c|c|c|c|c|c|c|c|}
\hline $\begin{array}{c}t_{\text {ann }} \\
{[\min ]}\end{array}$ & $\begin{array}{c}d_{\mathrm{d}-\mathrm{PS}} \\
{[\AA]}\end{array}$ & $\begin{array}{c}d_{\mathrm{h}-\mathrm{PMMA}}^{f} \\
{[\AA]}\end{array}$ & $\begin{array}{c}\rho_{\mathrm{h} \text {-PMMA }} \\
{\left[\begin{array}{ccc}10 & 6 & \AA\end{array}\right]}\end{array}$ & $\begin{array}{c}\rho_{\mathrm{d}-\mathrm{PS}} \\
{\left[\begin{array}{lll}10^{6} & { }^{6} & { }^{2}\end{array}\right]}\end{array}$ & $\begin{array}{c}\sigma_{\text {PS/PMMA }} \\
{[\AA \AA}\end{array}$ & $\begin{array}{c}\sigma_{\text {PMMA/Air }} \\
{[\AA]}\end{array}$ & $\begin{array}{c}\xi_{\mathrm{PMMA}} \\
{[\mu \mathrm{m}]}\end{array}$ & $\begin{array}{c}\xi_{\mathrm{PS}} \\
1 \mu \mathrm{mm}]\end{array}$ & $\begin{array}{c}\text { "PMMA/Air } \\
{[\mu \mathrm{m}]}\end{array}$ \\
\hline 0 & $107(1)$ & $3627(9)$ & $1.02(7)$ & $5.57(14)$ & $7.7(2)$ & $115(4)$ & $7.0(2)$ & $1.6(1)$ & $7.0(3)$ \\
\hline 5 & $103(1)$ & $3520(9)$ & $0.99(9)$ & $5.74(17)$ & $25.1(3)$ & $113(4)$ & $7.2(2)$ & $1.00(5)$ & $8.3(1)$ \\
\hline 10 & $117(1)$ & $3480(10)$ & $1.0(1)$ & $5.22(17)$ & $30.3(3)$ & $116(4)$ & $14.0(2)$ & $0.95(5)$ & $9.0(4)$ \\
\hline 15 & $133(1)$ & $3460(9)$ & $1.0(1)$ & $4.75(18)$ & $35.8(5)$ & $112(4)$ & $15.5(2)$ & $0.75(5)$ & $9.6(5)$ \\
\hline
\end{tabular}

Case B All fitting parameters for SR and OSS for the Case B sample.

Table S2

Fitted structural parameters for sample PMMA3500 as a function of annealing time. $\sigma_{\text {PSIFMMA }}$ is the Gaussian roughness between the d-PS and $h$-PMMA layers.

Case C All fitting parameters for SR and OSS for the Case C sample. 
Table S 3

Fitted structural parameter for sample PMMA BI as a function of annealing time. $\sigma_{\text {PSIMMMA }}$ is the Gaussian roughness between the $d-P S$ and $h$-PMMA layers.

\begin{tabular}{|c|c|c|c|c|c|c|c|}
\hline $\begin{array}{l}t_{\text {anth }} \\
{[\min ]}\end{array}$ & $\begin{array}{c}d_{\mathrm{d}-\mathrm{PS}} \\
{[\AA]}\end{array}$ & 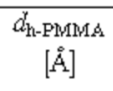 & 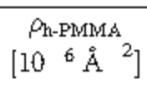 & 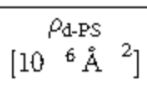 & $\begin{array}{c}\sigma_{\text {PSIPMMA }} \\
{[\stackrel{\AA}{\AA}]}\end{array}$ & $\begin{array}{c}\sigma_{\mathrm{PMMA} / / \mathrm{ir}} \\
{[\tilde{\AA}]}\end{array}$ & $\begin{array}{c}\xi_{P S I P M M A} \\
\lfloor\mu \mathrm{m}]\end{array}$ \\
\hline 0 & $114(1)$ & $2994(5)$ & $1.00(9)$ & $6.05(15)$ & $6.8(1)$ & $78(2)$ & $3.1(4)$ \\
\hline 5 & $111(1)$ & $2852(4)$ & $0.99(9)$ & $6.17(18)$ & $23.6(2)$ & $73(2)$ & $2.1(4)$ \\
\hline 10 & $111(1)$ & $2849(5)$ & $1.0(1)$ & $6.17(20)$ & $24.1(3)$ & $77(2)$ & $2.1(4)$ \\
\hline
\end{tabular}

